\title{
On the soft X-ray emission of M82
}

\author{
Jiren Liu ${ }^{1, \star}$, Q. Daniel Wang ${ }^{2}$, and Shude Mao ${ }^{1,3}$ \\ 1 National Astronomical Observatories, 20A Datun Road, Beijing 100012, China \\ 2 Department of Astronomy, University of Massachusetts, Amherst, MA 01002, USA \\ 3 Jodrell Bank Centre for Astrophysics, University of Manchester, Manchester, M13 9PL, UK
}

Received .., accepted ..

Published online later

Key words atomic processes - galaxies: individual (M82) - galaxies: starburst - X-rays: galaxies

\begin{abstract}
We present a spatial analysis of the soft X-ray and $\mathrm{H} \alpha$ emissions from the outflow of the starburst galaxy M82. We find that the two emissions are tightly correlated on various scales. The O VII triplet of M82, as resolved by X-ray grating observations of XMM-Newton, is dominated by the forbidden line, inconsistent with the thermal prediction. The O VII triplet also shows some spatial variations. We discuss three possible explanations for the observed O VII triplet, including the charge exchange at interfaces between the hot outflow and neutral cool gas, a collisional non-equilibrium-ionization recombining plasma, and resonance scattering.
\end{abstract}

(C) 0000 WILEY-VCH Verlag GmbH \& Co. KGaA, Weinheim

\section{Introduction}

Galactic-scale outflows (superwinds) from active star-forming galaxies represent an important feedback process, which regulates the galaxy evolution and recycles the metals and energy produced by massive stars and supernovae (Veilleux et at!. 2005). Indeed, $\mathrm{O}^{5+}$ ions have been detected out to $150 \mathrm{kpc}$ around star-forming galaxies by the Cosmic Origins Spectrograph through their absorption (Tumlinson et al. 2011). The details of the feedback process, however, can only be studied effectively through the X-ray emitting outflows of nearby starburst galaxies. The prototype starburst galaxy M82 (located at $3.6 \mathrm{Mpc}$ ), with a powerful superwind detected on scales up to $10 \mathrm{kpc}$, is an ideal target to study the physical process of superwind.

The soft X-ray emission of the outflow of M82 is found to be spatially correlated with the $\mathrm{H} \alpha$ emission (Strickland et al 2004), which indicates that the soft X-ray emission is due to the interaction between the hot outflow and the entrained disk/halo cool gas. While the shock-heated gas is expected to produce thermal soft $\mathrm{X}$-ray emission, the $\mathrm{O}$ VII triplet, as detected with XMM-Newton, is found to be dominated by the forbidden line (Liu et al. 2011; Ranalli et al. 2008), which is inconsistent with the thermal prediction.

The $\mathrm{K} \alpha$ triplet of He-like ions is a powerful diagnostic that can be used to test the origin of the X-ray line emission (for a recent review, see Porquet et al. 2010). The triplet consists of a resonance line, two inter-combination lines, and a forbidden line. For a thermal plasma in ionization equilibrium, the electron collisional excitation is efficient and favours the resonance line. The fact that the forbidden

\footnotetext{
* Corresponding author: e-mail: jirenliu@ nao.cas.cn
}

line is stronger than the resonance line suggests that an alternative emission mechanism may play a role in generating the soft X-ray emission.

One interesting possibility is the charge exchange (also called charge transfer), which occurs at the interface beat. een the hot plasma and neutral cool gas. It has been shown that highly ionised ions in the solar wind can readily pick up electrons from neutral species around a comet. These electrons, captured in excited states of the ions, cascade down and lead to X-ray line emission (e.g., Cravens 1997; Lisse et al. 1996). The electron downward cascading favours the forbidden line (for a recent review, see Dennerl 2010).

Similar interfaces between the hot plasma and cool gas also exists in the case of galactic superwinds. Observations have shown that the superwinds contain cool neutral and warm ionized clouds/filaments, as well as highly ionized hot ns. In the southwest outflow of M82 (see Fig. 1), molecular $\mathrm{H}_{2}$ and $\mathrm{CO}$ are observed to be well correlated with the $\mathrm{H} \alpha$ emission, thus with X-ray emission (Veilleux et al. 2009; Walter et al. 2002). The correlation between the molecular gas and the $\mathrm{H} \alpha$ emission is less prominent in the northeast outflow.

The relatively strong forbidden line of the $\mathrm{O}$ VII triplet of M82 may also be produced by the recombination of an overcooled non-equilibrium-ionization plasma. Another process that may have effect on the line ratio is the scattering of photons of a resonance line, which has a larger optical depth due to the large oscillator strength compared to a forbidden line.

In this paper we study the spatial correlation between the soft X-ray and $\mathrm{H} \alpha$ emissions using the high-resolution Chandra and HST data. We show that besides the global correlation between the soft X-ray and $\mathrm{H} \alpha$ emissions, there are regions showing that the soft X-ray emission occurs behind the $\mathrm{H} \alpha$ emission in the outflowing direction. We also 

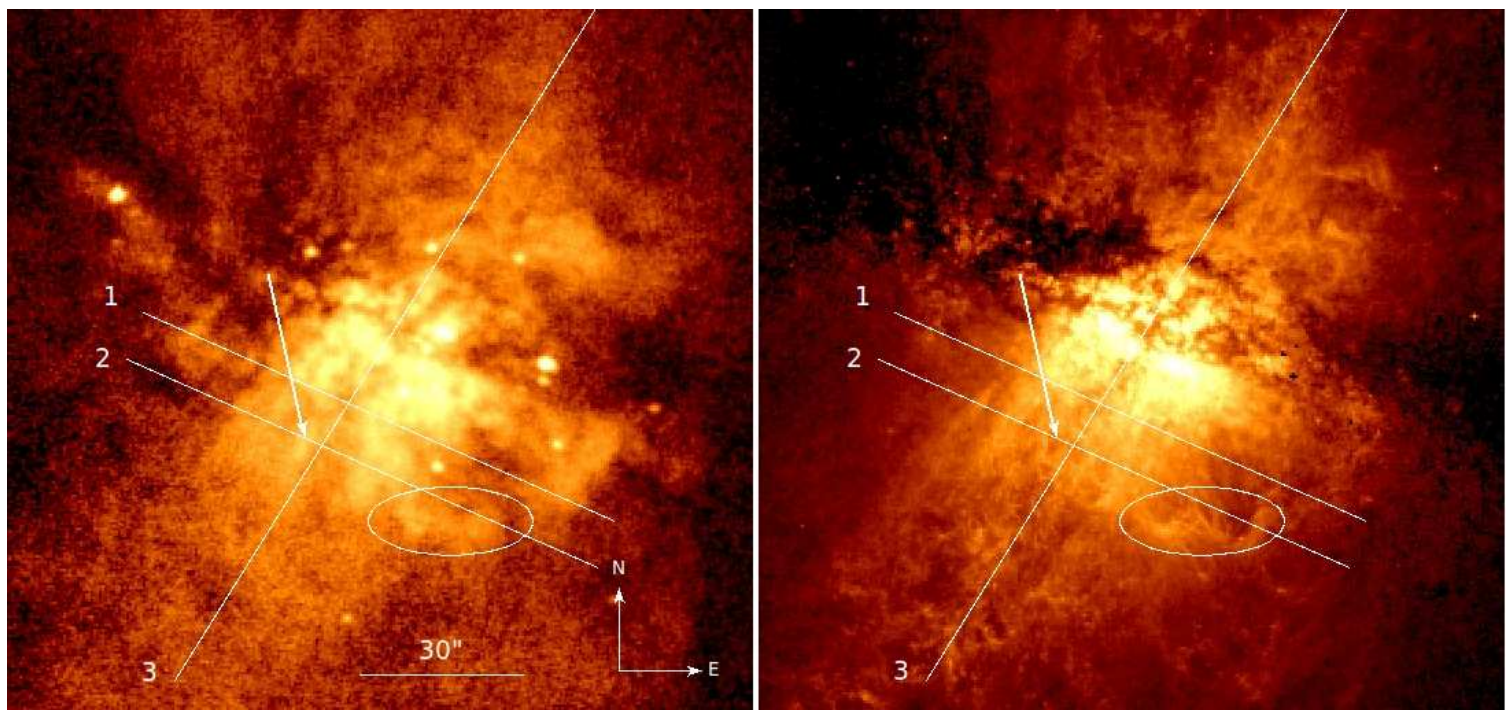

Fig. 1 Left: Chandra $0.5-2 \mathrm{keV}$ log-scaled intensity image of M82. Right: $H S T$ continuum subtracted H $\alpha$ emission of M82. Intensity distributions along the three marked lines are plotted in Fig. 2. The arrow and ellipse mark two regions that show close spatial connection between X-ray and $\mathrm{H} \alpha$ emissions.

present a spatially resolved analysis of the $\mathrm{O}$ vII triplet of M82. The three mechanisms mentioned above that can explain a strong forbidden line are discussed.

\section{Correlation between the soft $\mathrm{X}$-ray and $\mathrm{H} \alpha$ emissions}

The soft-X-ray emission ( $0.5-2 \mathrm{keV})$ of M82 observed by Chandra is plotted in Fig. 1 (left panel) together with the continuum subtracted $H S T \mathrm{H} \alpha$ image (right panel; Mutchler et al. 2007). The Chandra ACIS-S3 data are extracted from the observations with IDs of 10542, 10543, 10544, 10545, 10925, 11800 (PI: D. Strickland). The $H S T \mathrm{H} \alpha$ data, binned to $0.5^{\prime \prime}$ to match the pixel size of the Chandra image, shows many filamentary structures, as well as loops and arcs, while the X-ray image shows limb-brightening features. We see that in general, the morphology of X-ray emission follows that of $\mathrm{H} \alpha$ emission very well, though the $\mathrm{H} \alpha$ image shows much more detailed structures.

To illustrate their relation on finer scales, in Fig. 2, we plot the X-ray and $\mathrm{H} \alpha$ intensity distributions along three lines marked in Fig. 1. On large scales the profiles of both emissions follow with each other, but on small scales, there are regions showing distinctive features. For example, in the region marked by the ellipse in Fig. 1, the X-ray emission occurs behind the $\mathrm{H} \alpha$ emission in the outflowing direction (X-ray peak at the position 705 of line 2), and the $\mathrm{H} \alpha$ emission seems arising from shell-like structures driven by the $\mathrm{X}$-ray emitting outflow; at another region marked by the arrow, the peak of the X-ray filament coincides with faint $\mathrm{H} \alpha$ emission (X-ray peak at the position 640 of line 2).

Because of the projection effect, it is uncertain as to whether the large-scale similarity of the X-ray and $\mathrm{H} \alpha$ emis- sions is due to the superposition of small-scale adjacent features as shown along line 2 or due to the intrinsic association of two emission components, but the correlation indicates that the soft X-ray emission and $\mathrm{H} \alpha$ emission are closely connected.

\section{OVII triplet of $\mathrm{M82}$}

As stated in the introduction, the line ratios of the $\mathrm{O}$ VII triplet can be used to test the origin of the X-ray emission. To apply the test, the $\mathrm{O}$ VII triplet should be resolved. With its large dispersion power, XMM-Newton Reflection Grating Spectrometers (RGSs) (den Herder et al. 2001) have the unique capability to provide high resolution spectra for extended sources, such as the superwind of M82. We use two exposures with similar observational configurations (ID 0206080101 and 0560181301) and with a total effective exposure of 90 ks. To study the spatial behavior of the triplet, we divide the cross-dispersion range into three regions: A $\left(-30^{\prime \prime}-30^{\prime \prime}\right)$, B $\left(-90^{\prime \prime}--30^{\prime \prime}\right)$, and $C\left(30^{\prime \prime}-90^{\prime \prime}\right)$. Fig. 3 shows the dispersion direction and extraction regions plotted on the XMMNewton EPIC-pn image of M82.

The $\mathrm{O}$ VII triplets extracted from the three regions are plotted in Fig. 4. It can be seen clearly that the triplet of the $\mathrm{A}$ and $\mathrm{B}$ regions is dominated by the forbidden line. In contrast, the triplet is dominated by the resonance line in the $\mathrm{C}$ region. To study the line ratios of the $\mathrm{O}$ vII triplet quantitatively, we fit a model consisting of three Gaussians and a constant continuum to the data, which is expressed as

$f=\frac{1}{\sqrt{2 \pi} \sigma_{\lambda}} \sum_{j=\mathrm{r}, \mathrm{i}, \mathrm{f}} f_{j} \exp \left[-\frac{\left(\lambda-\lambda_{j}-\Delta \lambda\right)^{2}}{2 \sigma_{\lambda}^{2}}\right]+f_{\mathrm{con}},(1)$ where $\lambda_{\mathrm{r}, \mathrm{i}, \mathrm{f}}(21.6,21.8$ and $22.1 \AA)$ are the wavelengths of the resonance, inter-combination, and forbidden lines, re- 


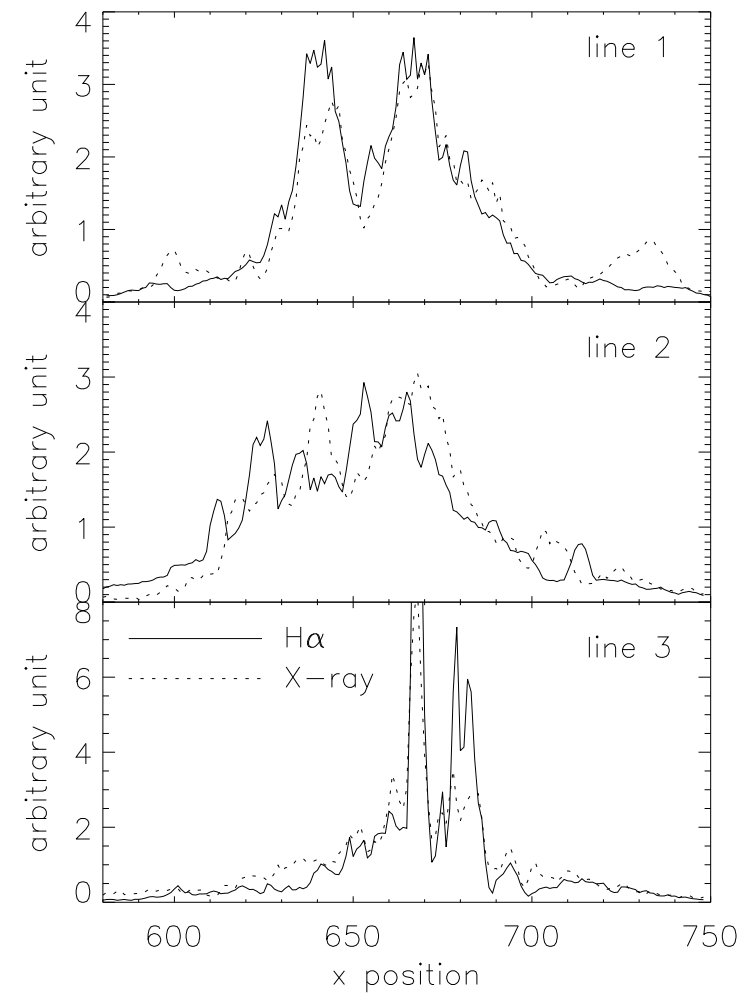

Fig. 2 The soft $\mathrm{X}$-ray and $\mathrm{H} \alpha$ intensity distributions along three specified lines marked in Fig. 1. The $\mathrm{X}$-axis is the horizontal pixel index of the image of Fig. 1 (with 1 pixel corresponding to $0.5^{\prime \prime}$ ).

spectively, $f_{\mathrm{r}, \mathrm{i}, \mathrm{f}}$ are the corresponding fluxes, $f_{\text {con }}$ is the continuum flux, $\sigma_{\lambda}$ the dispersion, and $\Delta \lambda$ the wavelength shift. The best-fitting results are plotted in Fig. 4 (for details, please see Liu et al. 2012).

For the emission from a thermal plasma in ionization equilibrium and at a temperature greater than $0.1 \mathrm{keV}$, the ratio $G=\frac{f_{\mathrm{f}}+f_{\mathrm{i}}}{f_{\mathrm{r}}}$ is smaller than 1 (e.g., Porquet et al. 2001). The $G$ ratios of A and B regions are around 3, inconsistent with the thermal prediction. The $G$ ratio in the $\mathrm{C}$ region is consistent with that of a thermal plasma around $5 \times 10^{6} \mathrm{~K}$.

\section{Discussions}

\subsection{Resonance scattering}

One possible explanation of the large $\mathrm{G}$ ratio is resonance scattering. If the optical depth is large enough, the intensity of the resonance line is re-distributed from the central optically thick region to the outer optically thin region (Gilfanov et al. 1987), and the ratio $G$ is increased in the central region and reduced in outer region. For the $\mathrm{A}$ and $\mathrm{B}$ regions, a diminishing factor of at least 3 is needed for the observed resonance line. If the relatively weak resonance line is indeed due to the resonance scattering, such a diminishment is also expected for other resonance lines, such as the $\mathrm{Fe}$ XVII $2 p-3 d$ line at $15 \AA$. Assuming the ratio of $\mathrm{Fe} / \mathrm{O}$ is

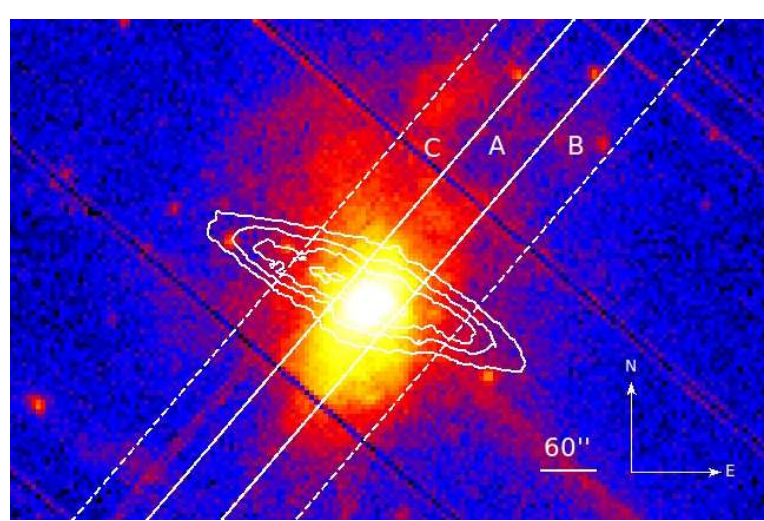

Fig. 3 Spectral extraction regions over-plotted on the soft X-ray (0.5-2 keV) XMM-Newton EPIC-pn image of M82. The presented lines outline the RGS dispersion direction and the regions (A, B, and C) from which the RGS spectra are exacted. Contours are from the DSS2 red image and indicate the disk of M82.

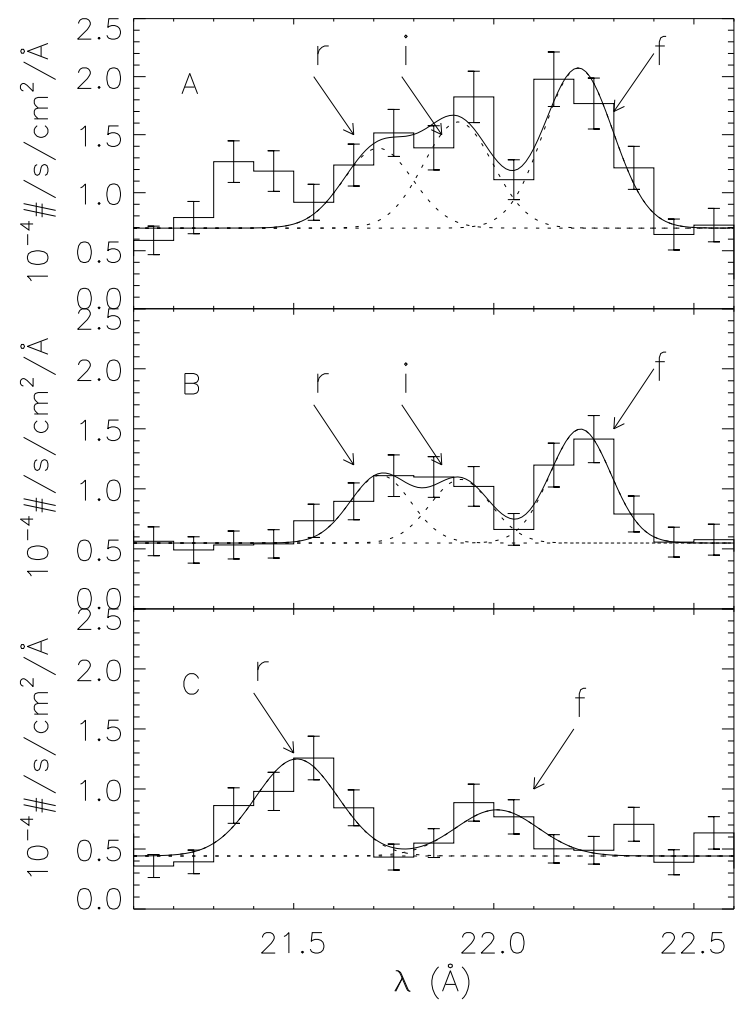

Fig. 4 RGS spectra of the O VII triplet for the three regions of M82 and the best-fit models (solid lines) of three Gaussians (dotted lines) and a constant continuum. The fitted resonance (r), inter-combination (i), and forbidden (f) lines are marked with arrows. 


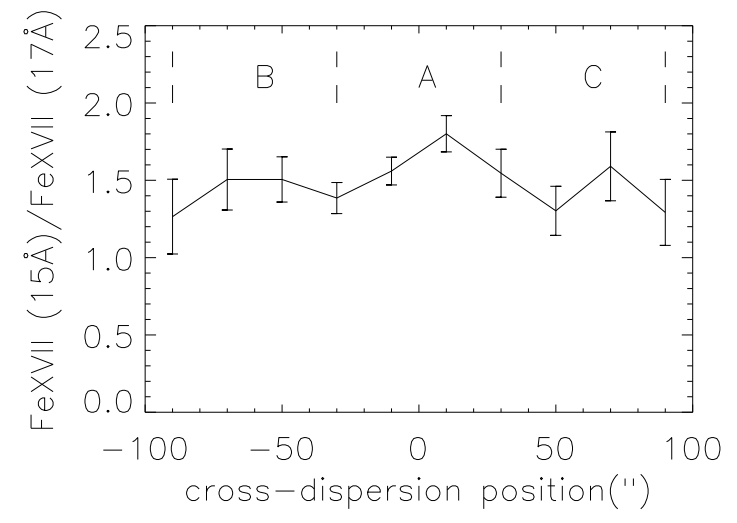

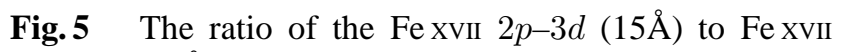
$2 p-3 s(17.1 \AA$ ) lines as a function of the cross-dispersion position. The three extraction regions shown in Fig. 3 are marked. The ratio is computed by selecting counts within a $0.2 \AA$ range centered on each line. As the RGS dispersion direction is not perpendicular to the outflow direction (Fig. 3 ), it leads to a wavelength-shift depending on the crossdispersion position. When computing the ratio we have corrected this effect.

similar to the solar value and a temperature around $3 \times 10^{6}$ $\mathrm{K}$ (estimated from the flux ratio of the $\mathrm{O}$ VIII Ly $\alpha$ to $\mathrm{O}$ VII triplet), the optical depth of the Fe XVII $2 p-3 d$ line is similar to that of the $\mathrm{O}$ VII resonance line. Because both the resonance Fe XVII $2 p-3 d$ line and the optically thin Fe XVII $2 p-3 s$ line at $17 \AA$ are relatively isolated, their ratio is a good test of the resonance scattering effect (Xu et al. 2002). In Fig. 5, we plot the cross-dispersion ratio of the Fe XVII $2 p-3 d$ line to the optically thin Fe XVII $2 p-3 s$ line. It shows little evidence for the diminishment of the $2 p-3 d$ line compared to the optically thin $2 p-3 s$ line, which argues against the resonance scattering effect.

\subsection{Non-equilibrium-ionization plasma}

A collisional non-equilibrium-ionization (NEI) recombining plasma can also produce a relatively strong forbidden line (e.g., Oelgoetz \& Pradhan 2004). A plasma, if expanding and cooling sufficiently fast, can become overly ionized. The recombination of the ions and electrons would then dominate the $\mathrm{X}$-ray line emission. However, the X-ray emission of M82 is spatially correlated with the filamentary $\mathrm{H} \alpha$ emission. This fact is consistent with the scenario that the $\mathrm{X}$-ray emission arises from the interfaces between the hot outflow and the entrained cool gas, but would be difficult to explain with the NEI model in which the emission should come from the bulk of the outflow, where the low density regions will be the most over-ionized. Furthermore, the A region shown in Fig. 3 is approximately the star-forming region, and the plasma there is hard to cool to low enough temperatures that the NEI condition can be achieved.

\subsection{Charge exchange $X$-ray emission}

The charge exchange $\mathrm{X}$-ray emission is the mechanism that can naturally explain the dominance of the forbidden line of the $\mathrm{O}_{\mathrm{VII}}$ triplet. It is supported by the tight correlation between the X-ray emission and the cool gas in the southwest outflow. The thermal-like $G$ ratio of the C region seems to arise from the northeast region with faint molecular gas (Veilleux et al. 2009). One spectral feature that can be used to further test the charge exchange $\mathrm{X}$-ray emission is the enhanced line flux from the levels $(n=3-6)$ of chargeexchange captured electrons, such as $\mathrm{O}$ VIII $\operatorname{Ly} \beta$ and $\operatorname{Ly} \gamma$ lines (Beiersdorfer et al. 2003). Another feature to test between the charge exchange and NEI model is the radiative recombination continuum (RRC), which is expected by the NEI model and not by the charge exchange. Unfortunately, current instruments do not have sufficiently large collecting area and high resolution to measure such spectral lines. With the calorimeter spectrometer of Astro-H planned to launch in 2014, one may be able to obtain a spatially resolved map of the $\mathrm{O}$ vil triplet, which could then be used to constrain the details of the charge exchange process.

\section{Acknowledgments}

We thank Thierry Montmerle and Rosine Lallement for organizing the CXU workshop and for their invitation and hospitality.

\section{References}

Beiersdorfer, P., Boyce, K. R., Brown, G. V., Chen, H., et al. 2003, Science, 300, 1558

Cravens, T. E. 1997, Geophys. Res. Lett., 24, 105

Dennerl, K. 2010, SSR., 157, 57

den Herder, J. W. et al. 2001, A\&A, 365, L7

Gilfanov, M. R., Syunyaev, R. A., Churazov, E. M. 1987, SvAL, 13, 3

Lisse, C. M., et al. 1996, Science, 274, 205

Liu, J., Mao, S., Wang, Q. D. 2011, MNRAS, 415, L64

Liu, J., Wang, Q. D., Mao, S. 2012, MNRAS, in press, arXiv: 1111.5915

Mutchler, M. et al. 2007, PASP, 119, 1

Oelgoetz, J. \& Pradhan, A. K. 2004, MNRAS, 354, 1093

Porquet, D., Mewe, R., Dubau, J., Raassen, A. J. J., Kaastra, J. S., 2001, A\&A, 376, 1113

Porquet, D., Dubau, J. \& Grosso, N. 2010, SSR., 157, 103

Ranalli, P., Comastri, A., Origlia, L., \& Maiolino, R. 2008, MNRAS, 386, 1464

Strickland, D. K., Heckman, T. M., Colbert, E. J. M., Hoopes, C. G., Weaver, K. A., 2004, ApJS, 151, 193

Tumlinson, J. et al. 2011, Sci, 334, 948

Veilleux, S., Cecil, G., Bland-Hawthorn, J. 2005, ARAA, 43, 769

Veilleux, S., Rupke, D. S. N., Swaters, R. 2009, ApJ, 700, L149 
Walter, F., Weiss, A., Scoville, N. 2002, ApJ, 580, L21

Xu, H., Kahn, S. M., Peterson, J. R., Behar, E. et al. 2002, ApJ, 579, 600 